\title{
Alcohol use and health care utilization in rural Liberia: Results of a community-based survey for basic public health indicators
}

\author{
Ana A. Weil ${ }^{1,2}$, Corey M. Cameron ${ }^{2,3}$, Jacob Soumerai ${ }^{1,2}$, Kerry L. Dierberg ${ }^{2,4}$, Alphonso Geelue Mouwon ${ }^{2}$, \\ David R. Kraemer, ${ }^{2,5}$, Daniela Lewy ${ }^{6}$, Patrick T. Lee ${ }^{1,2}$, John D. Kraemer, ${ }^{2,7}$, and Mark J. Siedner ${ }^{1,2}$ \\ ${ }^{1}$ Department of Medicine, Massachusetts General Hospital, Boston, MA, United States \\ ${ }^{2}$ Last Mile Health, Cambridge, MA, United States \\ ${ }^{3}$ Department of International Health, Georgetown University School of Nursing and Health Studies, Washington, DC, United States \\ ${ }^{4}$ Department of Medicine, The Johns Hopkins University School of Medicine, Baltimore, MD, United States \\ ${ }^{5}$ Department of Computer Studies, Kansas Wesleyan University, Salina, KS, United States \\ ${ }^{6}$ Department of International Health, Johns Hopkins Bloomberg School of Public Health, Baltimore, MD, United States \\ ${ }^{7}$ Department of Health Systems Administration, Georgetown University School of Nursing and Health Studies, Washington, DC, United States
}

\section{Abstract}

Aim: To measure the association between alcohol use and health-seeking behavior in post-conflict Liberia.

Design: Cross-sectional survey.

Setting: A community in rural southeast Liberia, from January 11 to January 16, 2010

Participants: 600 heads-of-household.

Measures: Logistic regression models for estimation of associations between alcohol use and indicators of healthcare utilization. Frequent alcohol use was defined as drinking more than seven days out of the last two weeks.

Findings: Frequent alcohol use was reported by $14.9 \%$ of participants. These respondents were less likely to attend clinic for chronic cough (Adjusted Odds Ratio, AOR 0.40, 95\% CI 0.18-0.87), to have had an HIV test (AOR 0.39, 95\% CI 0.19-0.77), and to have accessed prenatal care $(A O R=0.26,95 \%$ CI $0.12-0.54)$. Approximately $25 \%$ of all respondents had no access to latrines, and half reported going to sleep hungry in the past week.

Conclusions: Within households in post-conflict Liberia, there is an association between reduced health care utilization and frequent alcohol use self-reported by a head of household or primary caregiver.

Alcoholism, along with mental illness, is among the most neglected global health problems in the developing world (Bass et al., 2010; Patel, Goel, \& Desai, 2009). After a prolonged civil war, Liberia has the second lowest per capita GDP in the world (World Economic Outlook Database International Monetery Fund, 2011), and is recovering from widespread destruction of health infrastructure. Two decades of war, mass killings, forced conscription, and sexual violence have resulted in a population with a high risk of mental health disorders and alcohol abuse. In 2008, a survey of Liberian adults from across the country found that $40 \%$ met the symptom criteria for major depressive disorder and $44 \%$ for post-traumatic stress disorder (PTSD), and that ex-combatants were more likely to abuse alcohol than non-ex-combatants (Johnson et al., 2008). Another study of internally displaced persons in Liberia showed similarly high rates of alcohol and drug abuse (Ezard et al., 2011). Among forcibly displaced populations, risk factors for harmful alcohol use have been described, and include exposure to traumatic events, number of traumatic events endured, and resulting PTSD (de Jong et al., 2001; Weaver \& Roberts, 2010). Alcohol is a known contributor to poor health outcomes, including delayed diagnosis and treatment of conditions such as tuberculosis and HIV (de Albuquerque Mde et al., 2007; Samet et al., 1998; van der Werf et al., 2006; Waitt \& Squire, 2011). As a local non-governmental organization working in post-conflict Liberia, we posited that alcohol use was prevalent in our area, and might significantly impact health care utilization in our target population. The authors found that data specific to our catchment area on alcohol use and mental disorders, as well as basic health indicators, were not available. Our study aims to answer 
the following question: Is frequent alcohol use associated with differences in health care utilization compared to persons or households where alcohol is used less frequently?

Martha Tubman Memorial Hospital (MTMH) is in the city of Zwedru in Grand Gedeh County, which is among the poorest areas in Liberia, and home to refugees and migratory populations. Sources of national and regional data are informative but limited by convenience sampling and selection bias. In some areas, specific ethnic groups may not be willing to participate in demographic surveys, and rapidly changing refugee populations may make demographic assessment challenging. In resource-limited settings, there are published methods for collecting comprehensive health data (Demographic Health Surveys); however, these approaches are often too lengthy and expensive for use by small organizations operating at a community level. Using validated survey tools, we created the Community Healthy Living Survey (CHLS) in order to collect community-level health and demographic information to prioritize health-related activities.

\section{Method}

\section{Survey Design}

We developed the CHLS survey using questions about specific conditions that were previously validated (Coyne, Thombs, \& Mitchell, 2009; Doocy \& Burnham, 2006; Kullgren, Alibusa, \& Birabwa-Oketcho, 2009). We used methodology for basic epidemiologic surveys (The Epi Coverage Survey, 1991), favoring questions developed for resource-limited settings (Doocy \& Lewy, 2009) and targeted to priorities set by the Liberian Ministry of Health (Basic Package of Health and Social Welfare Services, 2008). Topics included alcohol use, malaria bednet ownership and use, childhood vaccination, prenatal service use, and screening questions for tuberculosis, sexually transmitted infections including HIV, depression, and food security. We included socioeconomic indicators, including access to health facilities, water and latrine sources (see Appendix 1). Chronic cough was defined as a cough lasting more than three weeks. Frequent alcohol use was defined by inquiring about norms of alcohol use in the local population with our Liberian counterparts, and was estimated by asking if alcohol had been consumed during at least seven days out of the previous two weeks.

\section{Interviewer Training}

Twelve community health workers (CHWs) with prior survey experience were recruited to perform the survey. CHWs participated in a two-hour review course on standardized survey methods, confidential survey administration, and acquisition of informed consent (Appendix 2). In addition, CHWs attended a four-hour training session on the CHLS tool and random cluster sampling methods.

\section{Cluster Selection}

The study population was that of the catchment area of MTMH. Due to frequent immigration, movement of people and outdated national census information, specific population data-including the total population for the area at the time of the survey-is not known. The study area included households within a two-hour walk of MTMH, and the sampling unit was the household. We selected a two-hour radius as the likely range of primary care service for rural residents without motorized transport. To select study villages, a blank 100-box grid was placed over the study area map. Using a random number table, we selected 30 numbers, and villages nearest to the grid boxes corresponding to those numbers were selected.

\section{Household and participant selection}

CHWs worked in pairs, and each pair surveyed 20 households per day. CHWs first located the village leader to introduce the study and request permission to proceed. Next, CHWs walked the perimeter of the village and drew a rough map of the area, divided into four numbered quadrants (Appendix 2). A number from one through four was randomly selected using four pieces of paper. CHWs walked to the center of the corresponding quadrant and spun a glass bottle or pen on the ground. Quadrants were used to divide the village into sections as a convenience method. The front door of the household nearest the direction of the bottle or pen was the first household surveyed. The household with the front door closest to the first household surveyed was chosen as the next household, and subsequent households were selected in the same manner until 20 households were surveyed in each cluster.

In order to select survey participants who were adults and were likely responsible for the care of others in their households, CHWs asked to speak to the primary caregiver or head of household. If this person was not at home, any other adult over age 17 was eligible to participate. Per Liberian law, minors are persons aged 16 and younger; therefore, participants 17 and older were considered adults. When no adults were home, the household was revisited twice during the study day. If an eligible adult could not be identified after three visits to a household, the household was omitted and an additional household was selected by the same manner as subsequent households were identified above. A member of a household was defined as a person who has slept in the same dwelling and eaten out of the same cooking pot for the previous three weeks.

Once a household was selected, CHWs introduced the study to potential participants. CHWs traveled as malefemale pairs and same-sex interviews were conducted when possible. CHWs explained the study and obtained verbal informed consent after identifying a private location. For survey respondents with acute health needs identified during survey administration, CHWs gave MTMH hospital referral information. After the survey was conducted, all respondents were informed of the free services available at MTMH, including HIV testing, antenatal clinics (ANC), and treatment programs for epilepsy, depression, and HIV. All surveys were conducted between January 11 and January 16, 2010. At the time of the study, the Liberian 
human subjects ethics review committee was under development. The institutional review board of Massachusetts General Hospital and local administrative officials in Liberia approved use of the data presented in this paper for research purposes.

\section{Sample size calculation}

With a goal of estimating disease prevalence at $\pm 5 \%$, and considering our cluster design, we estimated a goal sample size of 540 households. To account for irregularities in survey administration, we aimed to survey 600 individuals.

\section{Statistical methods}

Summary statistical methods were used to describe demographic, socio-economic, and clinical characteristics. Differences between groups were compared using logistic regression. Standard errors were adjusted for the clustered design using Taylor-linearized variance estimation in all analyses. When outcome variables were continuous, differences in median values were tested using Somers' D test for rank across ordered variables, taking into account the clustering of the data.

Our primary predictor of interest was frequent alcohol use, defined as respondent report of alcohol consumption on more than half of days. The volume of alcohol use was not collected. For each of our measured outcomes, we fit both univariable and multivariable regression models and adjusted for respondent sex, age, geographic location (residence within 20-minute walk of the town center), and cell phone ownership. Because these factors were hypothesized to be putative predictors of accessing services, all were retained in the multivariate model, except for sex, which was only included when the outcome related to the respondent (as opposed to the respondent's household). A 20-minute cutoff approximated the distance threshold between those living in or near town and those living more distantly, and the authors found that inclusion of distance as a continuous variable detracted from the statistical model's power without improving goodness of fit; therefore, a binary variable was used. Five hundred and twenty participants were included in the multivariate analysis. Because patterns of alcohol use vary by sex globally, we tested for interactions between frequent alcohol use and the respondents' sex; however, the interaction terms were dropped from the multivariate models because they did not improve the models' goodness of fit based on Akaike Information Criterion and because no interaction coefficients were statistically significant.

Odds ratios (ORs) with 95\% confidence intervals (95\% CIs) are reported. A cutoff of $p<0.05$ was the predetermined threshold for statistical significance. All reported $p$-values are 2-tailed. Microsoft Access was used for data storage (contact the corresponding author for a free, editable version of the database). Analyses were performed on GraphPad Prism 4.0 and Stata version 11.2 (Stata Corp., 2009).

\section{Results}

We surveyed a total of 600 respondents from 20 households in 30 clusters. Eleven households did not have an adult available to complete the study. Zero respondents refused to participate. CHWs conducted the surveys in a total of six days, at a rate of 100 households per day, and interviews lasted approximately 15 minutes on average. The physical area where the survey was conducted was not measured. Due to omitted questions, denominator numbers for responses vary. Although the survey included a depression screening tool, these results were discarded due to the high level of inter-interviewer variability of question delivery observed by study coordinators. The total cost for the CHLS study was $\$ 949.00$ USD (\$1.58 per household surveyed). Ten CHWs and two CHW supervisors were paid \$30.00 for working six days, consistent with local standards of pay. Survey materials, training lunch and office supply costs totaled \$589.00. Organization volunteers conducted data entry, data analysis, and questionnaire and training manual development. There were no transportation costs.

Most respondents were female (345/529, or $65 \%$ ) and the mean age was 41 years (range 17-98) (Table 1). The median household size was seven members (interquartile range [IQR] 5-10), with a median of two children under five (IQR 1-3). Forty-eight percent of all households reported ownership of one or more bednets (285/594). Of the 1,209 children over five years in 590 households, $30 \%$ (350/1209) slept under a bednet the night prior to survey. Sixty percent of children over five years (339/565) had full vaccination coverage, as measured by reported receipt of five vaccinations in the first year of life.

Among 17- to 49-year-olds, 207 out of 441 (47\%) reported a previous HIV test. Some respondents were not aware of their testing results, and four (4/196, or $2.4 \%$ ) reported a positive result. One respondent over the age of 49 years reported a positive HIV test result. Twenty-six percent of respondents aged 17 to 49 reported symptoms of a sexually transmitted disease (abnormal discharge or sores/lesions in the genital area) during the previous six months. Twentyfive percent (146/590) of respondents reported cough for the preceding three weeks. Sixty percent $(77 / 127)$ of these individuals sought care at a health center.

Fifteen percent of respondents (85/569) reported frequent drinking, defined as alcohol consumption on at least seven days of the previous two weeks. Frequent drinking was more common in male respondents $(22.4 \%)$ than female (10.1\%), and this difference was statistically significant ( $p$ $=0.002)$. Of the five participants who reported positive HIV test results, only four answered the question about alcohol use, and none reported frequent alcohol use. Half of respondents reported going to bed hungry at least once in the last week (275/549, or 50\%). Half of respondents reported cell phone ownership (276/576, or 49\%). Cell phone ownership was significantly more likely among respondents who lived a 20-minute walk or less from the town center than among those who lived more than a 20minute walk from the town center $(O R=2.5$, CI 1.5-4.2). 
Table 1

Demographic, socioeconomic, and clinical characteristics

\begin{tabular}{|c|c|c|c|c|}
\hline Survey Question & $\begin{array}{l}\text { Drinks Alcohol } \geq 7 \text { days } \\
\text { in a 14-day period }\end{array}$ & $\begin{array}{l}\text { Drinks Alcohol }<7 \text { days } \\
\text { in a 14-day period }\end{array}$ & Total & $P$ value \\
\hline Female sex & $33 / 73(45 \%)$ & $293 / 432(68 \%)$ & $345 / 529(65 \%)$ & $<0.01002$ \\
\hline Median household size IQR & 7.5 members (6-10) & 7 members (5-10) & 7 members (5-10) & 0.187 \\
\hline $\begin{array}{l}\text { Living within } 20 \text {-min walk of town } \\
\text { center }\end{array}$ & 43/85 (51\%) & $290 / 484(60 \%)$ & $357 / 596(60 \%)$ & 0.312 \\
\hline Cell phone ownership & $43 / 83(51 \%)$ & $229 / 470(49 \%)$ & $280 / 576(49 \%)$ & 0.738 \\
\hline $\begin{array}{l}\text { Defecates in open field no toilet or } \\
\text { latrine }\end{array}$ & $10 / 84(12 \%)$ & $109 / 474(23 \%)$ & $124 / 580(21 \%)$ & 0.025 \\
\hline Unprotected water source & $16 / 81(20 \%)$ & $106 / 470(23 \%)$ & $126 / 574(22 \%)$ & 0.656 \\
\hline Metal house roof & $32 / 84(38 \%)$ & $181 / 482(38 \%)$ & $218 / 569(37 \%)$ & 0.977 \\
\hline Chronic cough & 28/85 (33\%) & $112 / 479(23 \%)$ & $146 / 590(25 \%)$ & 0.039 \\
\hline Recent penile/vaginal discharge or sore & $20 / 81(25 \%)$ & $102 / 479(21 \%)$ & $126 / 583$ (22\%) & 0.449 \\
\hline Household bednet ownership & $57 / 85(67 \%)$ & $219 / 482$ (45\%) & $276 / 567$ (49\%) & 0.003 \\
\hline Complete vaccination & $40 / 81(49 \%)$ & $288 / 460(63 \%)$ & $328 / 541(61 \%)$ & 0.079 \\
\hline Prior HIV test & $21 / 84(25 \%)$ & $199 / 480(41 \%)$ & $220 / 564(39 \%)$ & 0.006 \\
\hline
\end{tabular}

Due to omitted questions, denominator numbers for responses vary. Differences between groups were compared using chi-squared testing. Median age and household size were tested using Somers' D, taking into account the clustered nature of the data. All other comparisons use Pearson's chi-square, adjusted for the clustered nature of the data.

Table 2a

Logistic regression of HIV testing on alcohol use and predictor variables

\begin{tabular}{|c|c|c|c|c|c|}
\hline & \multicolumn{3}{|c|}{ Bivariate } & \multicolumn{2}{|c|}{ Multivariate $(n=480)$} \\
\hline & Crude OR (95\% CI) & $\boldsymbol{P}$ & $n$ & Adjusted $O R(95 \% \mathrm{CI})$ & $\boldsymbol{P}$ \\
\hline Alcohol use $>1 / 2$ days & $0.47(0.28-0.80)$ & 0.007 & 564 & $0.39(0.19-0.77)$ & 0.009 \\
\hline Female & $1.5(0.94-2.4)$ & 0.090 & 520 & $1.4(0.87-2.2)$ & 0.176 \\
\hline Living within 20 -min walk of town center & $1.7(1.1-2.4)$ & 0.010 & 587 & $1.0(0.59-1.7)$ & 0.997 \\
\hline Cell phone ownership & $1.4(0.93-2.0)$ & 0.103 & 572 & $1.5(0.93-2.3)$ & 0.098 \\
\hline Age (per 1-yr increase) & $0.96(0.94-0.97)$ & $<0.001$ & 575 & $0.96(0.94-0.98)$ & $<0.001$ \\
\hline
\end{tabular}

Multiple logistic regresssion with standard errors adjusted for the clustered design using Taylor-linearized variance estimation was used to generate this data.

Table 2b

Logistic regression of clinic attendance among those with chronic cough on alcohol use and other predictor variables

\begin{tabular}{|c|c|c|c|c|c|}
\hline & \multicolumn{3}{|c|}{ Bivariate } & \multicolumn{2}{|c|}{ Multivariate $(n=103)$} \\
\hline & Crude OR (95\% CI) & $\boldsymbol{P}$ & $n$ & Adjusted OR (95\% CI) & $\boldsymbol{P}$ \\
\hline Alcohol use $>1 / 2$ days & $0.48(0.24-0.98)$ & 0.044 & 123 & $0.40(0.18-0.87)$ & 0.027 \\
\hline Female & $1.8(0.85-3.8)$ & 0.123 & 109 & $2.3(1.1-4.8)$ & 0.031 \\
\hline Living within 20 -min walk of town center & $1.2(0.63-2.4)$ & 0.513 & 127 & $0.77(0.34-1.8)$ & 0.523 \\
\hline Cell phone ownership & $2.1(1.1-4.0)$ & 0.028 & 125 & $2.8(1.2-6.5)$ & 0.020 \\
\hline Age (per 1-yr increase) & $1.0(0.98-1.0)$ & 0.775 & 126 & $1.0(0.99-1.0)$ & 0.686 \\
\hline
\end{tabular}

Multiple logistic regression with standard errors adjusted for the clustered design using Taylor-linearized variance estimation was used to generate this data. 
Table 2c

Logistic regression of antenatal clinic attendance among respondents who are pregnant or who have pregnant partners on alcohol use and other predictor variables

\begin{tabular}{|c|c|c|c|c|c|}
\hline & \multicolumn{3}{|c|}{ Bivariate } & \multicolumn{2}{|c|}{ Multivariate $(n=70)$} \\
\hline & Crude OR (95\% CI) & $\boldsymbol{P}$ & $n$ & Adjusted $O R(95 \% \mathrm{CI})$ & $\boldsymbol{P}$ \\
\hline Alcohol use $>1 / 2$ days & $0.25(0.14-0.45)$ & $<0.001$ & 71 & $0.26(0.12-0.54)$ & 0.001 \\
\hline Living within 20 -min walk of town center & $1.4(0.45-4.4)$ & 0.545 & 74 & $1.6(0.59-4.6)$ & 0.327 \\
\hline Cell phone ownership & $0.65(0.26-1.6)$ & 0.348 & 74 & $0.50(0.19-1.3)$ & 0.162 \\
\hline Age (per 1-yr increase) & $1.0(0.95-1.1)$ & 0.829 & 73 & $1.0(0.96-1.1)$ & 0.593 \\
\hline
\end{tabular}

Multiple logistic regression with standard errors adjusted for the clustered design using Taylor-linearized variance estimation was used to generate this data.

Adjusting for other factors, frequent alcohol use was significantly associated with poor health-seeking behaviors. In multivariable logistic regression models, frequent drinkers had decreased odds of having had an HIV test (Adjusted Odds Ratio, $A O R=0.39, p=0.009$ ) or seeking care if they had a chronic cough $(A O R=0.40, p=0.0303)$. Frequent drinkers who were pregnant or had a pregnant partner were less likely to have accessed prenatal care $(A O R=0.26, p=0.001$, Tables 2a-c). Frequent drinkers were less likely to report going to bed hungry in the last week $(A O R=0.53, p=0.0016)$ and their households were more likely to own at least one bednet $(A O R=2.3, p<$ 0.004).

\section{Discussion}

While direct health impacts are not measured in this study, an association exists between frequent alcohol use in Liberian adults and specific health indicators. This possibility is consistent with other studies, where alcohol use has been associated with nearly $4 \%$ of all-cause mortality; furthermore, the attributable disease burden is most prominent among marginalized populations (Casswell \& Thamarangsi, 2009; Rehm et al., 2009). Many existing studies of alcohol use focus on the effects of immediate use, such as reduced medication adherence, accidental injury, gender-based violence, and sexual behaviors. More recently, the effects of alcohol use on chronic disease and mortality have been recognized (Rehm \& Parry, 2009). Our study suggests that there is an association between frequent alcohol use and measures of household health.

We found that individuals who did not report frequent alcohol use and younger individuals were more likely to have been tested for HIV. Consistent with this finding, a study of South African women found that alcohol consumption was linked to lower rates of HIV testing (Luseno \& Wechsberg, 2009). In our study, the prevalence of self-reported HIV infection was low, and consistent with known national HIV estimates for Liberia, with a reported prevalence of $1.5 \%$ among 15 - to 49-year-olds estimated in 2007 by the Demographic and Health Survey (HIV/AIDS
Data from the 2007 Liberia Demographic and Health Survey, 2007). Because HIV was uncommon in our cohort, it was not possible to draw conclusions about the relationship between HIV infection and frequent alcohol use.

In the last decade, recognition of alcohol use and mental illness as neglected public health issues in low-resource countries has led to a call for action (Patel et al., 2009). In Liberia, several studies estimate a high prevalence of mental illness (Galea et al., 2010; Johnson et al., 2008). Alcoholism is frequently co-morbid with depression and PTSD, and this has been found to be characteristic of other African post-conflict populations (Okulate \& Jones, 2006). Appreciation for the scope of this problem has prompted the Liberian government to initiate mental health service provision nationwide over the next decade (Lee et al., 2011). Our results suggest a need for further studies to explore social mores and cultural reasons for alcohol use, to characterize alcohol use versus abuse in Liberia, and to assess knowledge about the harms of frequent alcohol use.

There are limitations to our survey design. Our CHW training was brief, which may have influenced the integrity of the data. A pilot survey or a back-translation of the survey in the specific local Liberian English dialect might have improved comprehension and precision of survey results. For example, we removed results for the PHQ-2 depression screen from our analysis after learning of differing interpretation of questions in the local language by CHWs. The authors would recommend a more extensive training session for interviewers in future studies. An assessment of inter-rater reliability would enhance integrity of data in future studies. The brevity of our survey likely led to a failure to address confounders, including mental disorders. The authors also did not use a validated tool for alcohol consumption, such as AUDIT or CAGE, which could have significantly increased the validity and reliability of these results. Other assessment tools created for assessments in conflict-affected populations could have been used (Ezard et al., 2011). Our definition of frequent alcohol use did not include the type of alcohol or volume consumed, or the pattern of drinking. There are guidelines for accepted levels of alcohol 
consumption, and individuals drinking alcohol on half of the days in a two-week period may not have reached an inappropriate level of alcohol use according to many of these guidelines; however, there is disagreement about safe levels of alcohol consumption (Furtwaengler \& de Visser, 2012; Stockwell, Butt, Beirness, Gliksman, \& Paradis, 2012). The variable for alcohol consumption of drinking on more than half of the days in a two-week period was based on information from local counterparts about norms of alcohol consumption, and does not necessarily reflect an unsafe level of consumption. More females than males responded to our survey, which may have resulted in an underreporting of alcohol use, since men are known to consume more alcohol than women in most low-income countries (Room \& Obot, 2008). It is also possible that respondents minimized their alcohol consumption when reporting to health care workers. Socioeconomic and educational data on our participants was not gathered; this information may have revealed important confounders that could have influenced the interpretation of results. The head of household or primary caregiver was self-identified or identified by other household members, instead of being defined using an objective metric, which may have introduced bias; for example, answers to the food security question, "How many days did you go to sleep hungry last week?”, might not equally represent all household members. Surveying the primary caregiver or household head does not represent all adults in the population, and the number of other adults in the household surveyed because the head of household was unavailable was not recorded. In addition, the cluster sampling method may have led to a cluster effect and introduced bias. Some respondents did not answer all questions in the survey, and the nonresponse rate compromised the data quality, notably in the multivariate analysis, where only 520 respondents' responses were used.

In summary, we found that in post-conflict Liberia, there is an association between reduced health care utilization and frequent alcohol use self-reported by a head of household or primary caregiver. Further research is needed to explore the relationship between frequent alcohol use and primary care utilization.

\section{Acknowledgements}

We are grateful for the participation of the people of Grand Gedeh County, Liberia. We thank the community health workers of the Martha Tubman Memorial Hospital and Last Mile Health. We also thank Dr. Hanni Stoklosa for logistical assistance and Dr. Namah Shah for sampling methodology technical assistance. The authors declare that they have no conflicts of interest or financial disclosures.

\section{References}

Basic Package of Health and Social Welfare Services. (2008). Ministry of Health and Social Welfare, Republic of Liberia. Retrieved from http://www.
basics.org/documents/Basic-Package-of-Health-andSocial-Welfare-Services_Liberia.pdf.

Bass, J. K., Bornemann, T. H., Burkey, M., Chehil, S., Chen, L., Copeland, J. R., ... Patel, V. (2010). A United Nations General Assembly Special Session for mental, neurological, and substance use disorders: The time has come. PLoS Medicine, 9, e1001159.

Casswell, S., \& Thamarangsi, T. (2009). Reducing harm from alcohol: Call to action. Lancet, 373, 2247-2257.

Coyne, J. C., Thombs, B. D., \& Mitchell, A. J. (2009). PHQ-9 and PHQ-2 in Western Kenya. Journal of General Internal Medicine, 24, 890.

de Albuquerque Mde. F., Ximenes, R. A., Lucena-Silva, N., de Souza, W. V., Dantas, A. T., Dantas, O. M., \& Rodrigues, L. C. (2007). Factors associated with treatment failure, dropout, and death in a cohort of tuberculosis patients in Recife, Pernambuco State, Brazil. Cadernos de Saude Publica, 23, 1573-1582.

de Jong, J. T., Komproe, I. H., Van Ommeren, M., El Masri, M., Araya, M., Khaled, N., . . Somasundaram, D. (2001). Lifetime events and posttraumatic stress disorder in 4 postconflict settings. JAMA, 286, 555562.

Demographic Health Surveys. (Key Indicator Surveys.) http://www.measuredhs.com/aboutsurveys/kis.cfm

Doocy, S., \& Burnham, G. (2006). Assessment of socioeconomic status in the context of food insecurity: Implications for field research. World Health Population, 8, 32-42.

Doocy, S., \& Lewy, D. (2009). Mortality and nutrition in Lofa County, Liberia: Johns Hopkins Bloomberg School of Public Health, International Medical Corps. Retrieved from http://www.internationalmedicalcorps. org/Document.Doc?id=23

Ezard, N., Oppenheimer, E., Burton, A., Schilperoord, M., Macdonald, D., Adelekan, M., ... van Ommeren, M. (2011). Six rapid assessments of alcohol and other substance use in populations displaced by conflict. Conflict Health, 5, 1.

Furtwaengler, N. A., \& de Visser, R. O. (2012). Lack of international consensus in low-risk drinking guidelines. Drug and Alcohol Review, 32, 11-18. doi:10.1111/j.1465-3362.2012.00475.x

Galea, S., Rockers, P. C., Saydee, G., Macauley, R., Varpilah, S. T., \& Kruk, M. E. (2010). Persistent psychopathology in the wake of civil war: Long-term posttraumatic stress disorder in Nimba County, Liberia. American Journal of Public Health, 100, 1745-1751.

HIV/AIDS Data from the 2007 Liberia Demographic and Health Survey. (2007). Retrieved from http://www. measuredhs.com/publications/publication-hf24-hivfact-sheets.cfm

Johnson, K., Asher, J., Rosborough, S., Raja, A., Panjabi, R., Beadling, C., \& Lawry, L. (2008). Association of combatant status and sexual violence with health and mental health outcomes in post conflict Liberia. JAMA, 300, 676-690.

Kullgren, G., Alibusa, S., \& Birabwa-Oketcho, H. (2009). Problem drinking among patients attending primary healthcare units in Kampala, Uganda. African Journal of Psychiatry, 12, 52-58. 
Lee, P. T., Kruse, G. R., Chan, B. T., Massaquoi, M. B., Panjabi, R. R., Dahn, B. T., \& Gwenigale, W. T. (2011). An analysis of Liberia's 2007 national health policy: Lessons for health systems strengthening and chronic disease care in poor, post-conflict countries. Global Health, 7, 37.

Luseno, W. K., \& Wechsberg, W. M. (2009). Correlates of HIV testing among South African women with high sexual and substance-use risk behaviours. AIDS Care, $21,178-184$.

Okulate, G. T., \& Jones, O. B. (2006). Post-traumatic stress disorder, survivor guilt and substance use: A study of hospitalised Nigerian army veterans. South African Medical Journal, 96, 144-146.

Patel, V., Goel, D. S., \& Desai, R. (2009). Scaling up services for mental and neurological disorders in lowresource settings. International Health, 1, 37-44.

Rehm, J., Mathers, C., Popova, S., Thavorncharoensap, M., Teerawattananon, Y., \& Patra, J. (2009). Global burden of disease and injury and economic cost attributable to alcohol use and alcohol-use disorders. Lancet, 373, 2223-2233.

Rehm, J., \& Parry, C. (2009). Alcohol consumption and infectious diseases in South Africa. Lancet, 374, 2053.

Obot, I. S., \& Room R, (2008). Alcohol, gender and drinking problems. Perspectives from low and middle income countries. World Health Organization, Department of Mental Health and Substance Abuse: Geneva, Switzerland.

Samet, J. H., Freedberg, K. A., Stein, M. D., Lewis, R., Savetsky, J., Sullivan, L., ... Hingson, R. (1998). Trillion virion delay: Time from testing positive for
HIV to presentation for primary care. Archives of Internal Medicine, 158, 734-740.

StataCorp. 2009. Stata 11 Base Reference Manual. College Station, TX, United States: Stata Press.

Stockwell, T., Butt, P., Beirness, D., Gliksman, L., \& Paradis, C. (2012). The basis for Canada's new lowrisk drinking guidelines: A relative risk approach to estimating hazardous levels and patterns of alcohol use. Drug and Alcohol Review, 31, 126-134.

The Epi Coverage Survey. (1991). World Health Organization expanded programme on immunization. Retrieved from http://www.who.int/immunization_ monitoring/routine/EPI_coverage_survey.pdf

van der Werf, M. J., Chechulin, Y., Yegorova, O. B., Marcinuk, T., Stopolyanskiy, A., Voloschuk, V., ... Turchenko, L. V. (2006). Health care seeking behaviour for tuberculosis symptoms in Kiev City, Ukraine. International Journal of Tuberculosis and Lung Disease, 10, 390-395.

Waitt, C. J., \& Squire, S. B. (2011). A systematic review of risk factors for death in adults during and after tuberculosis treatment. International Journal of Tuberculosis and Lung Disease, 15, 871-885.

Weaver, H., \& Roberts, B. (2010). Drinking and displacement: A systematic review of the influence of forced displacement on harmful alcohol use. Substance Use and Misuse, 45, 2340-2355.

World Economic Outlook Database International Monetery Fund. (2011). Retrieved from www.imf.org/external/ pubs/ft/weo/2011/01/weodata/index.aspx 


\section{Appendices}

\section{Appendix 1}

Community Healthy Living Survey and Consent

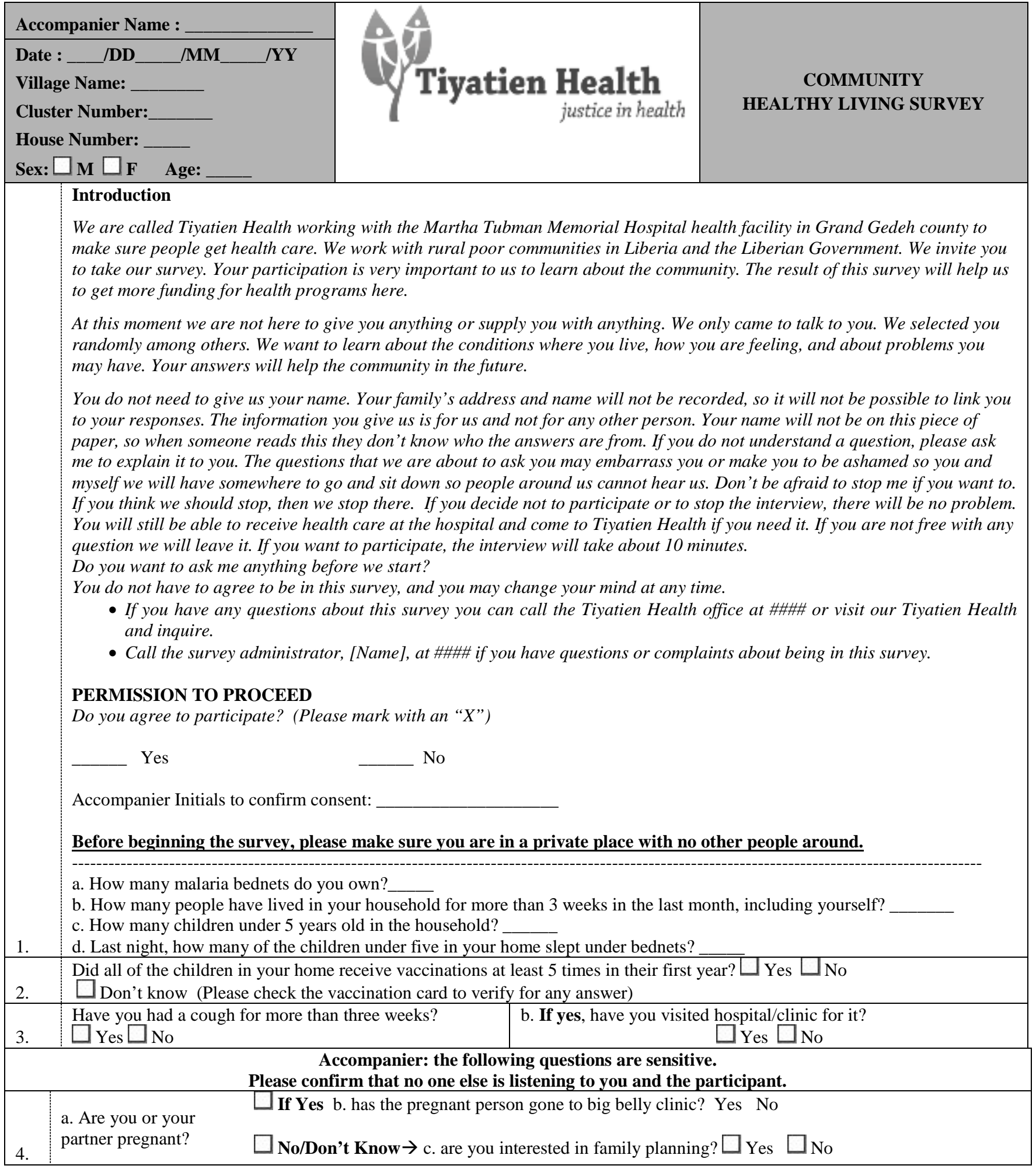




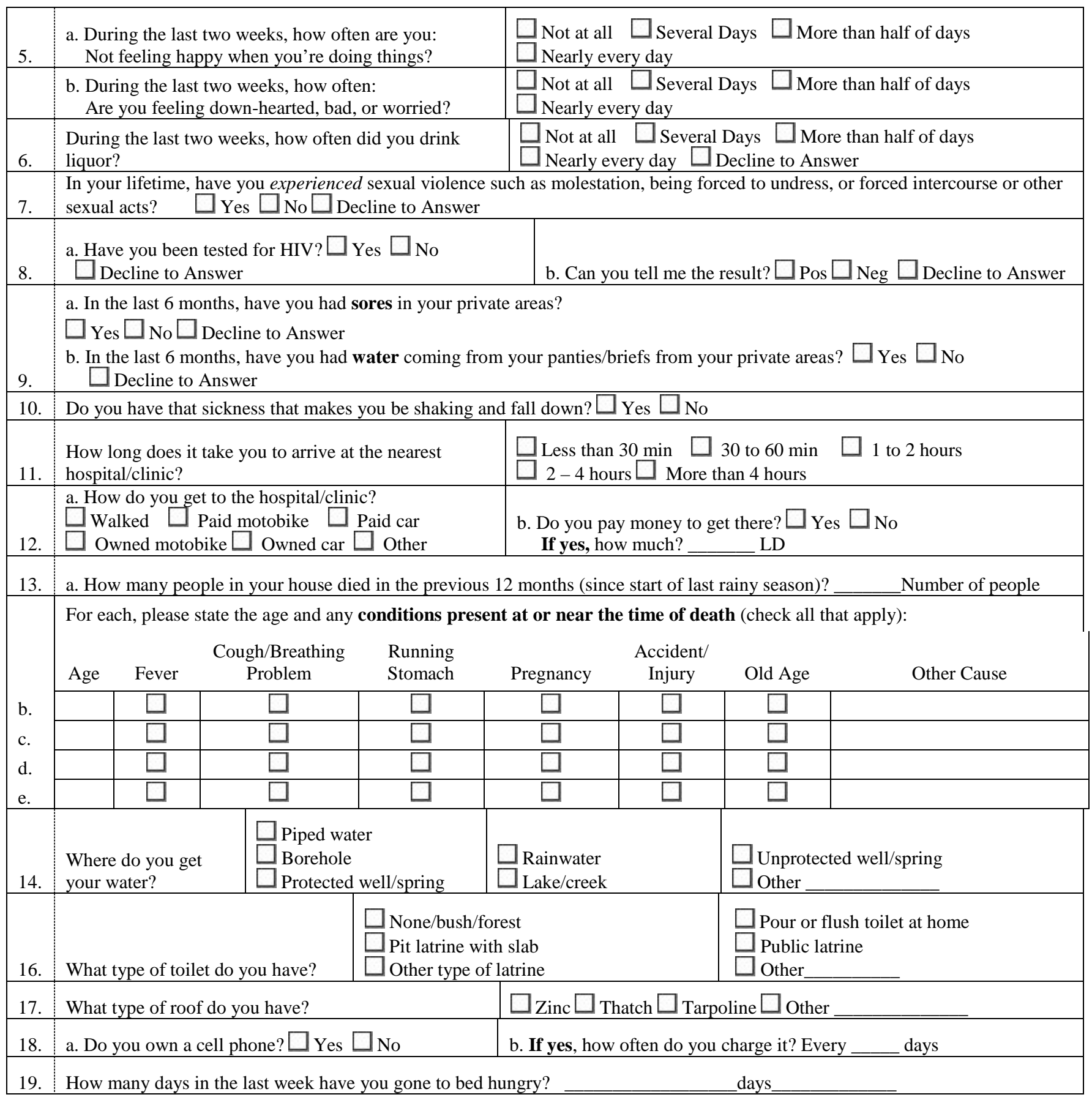

Closing and self-referral

Thank you so much for your participation in this survey. Tiyatien Health hopes to use the information you provided about your experiences to inform our health programs. If you, your family, or anyone in your community would benefit from our health services, please come to our clinic. Thank you again for your help today.

Before leaving, please give specific information about referrals. Please remember Tiyatien and MTMH programs including VCT, antenatal care, HIV, epilepsy and depression. 


\section{Appendix 2}

\section{Community Health Living Survey (CHLS) Training Materials}

\section{What is the purpose of the survey?}

Thank you for participating in the CHLS! This survey will be done to find out what the health needs are near Martha Tubman Memorial Hospital. With this information, Tiyatien Health will create programs to help people in the future.

We are excited about gathering information from the community in order to improve clinical programs and secure more funding. We appreciate your help in gathering this information. We hope this survey is the first step to making change in Zwedru and living healthy lives in our community.

This is a guide to help persons conducting the survey to carry it out correctly. A survey not done correctly wastes time and money that many people spent, and if done incorrectly money and efforts for helping people will be directed toward wrong activities.

\section{What type of survey is this?}

This survey is a 'cluster survey.' Each 'cluster' consists of 20 households which are located close to each other. The entire survey consists of 30 clusters, or 600 households.

Accompaniers will work in pairs. Each day, each pair must interview 20 households in a cluster in each location so they do not have to return to that place on the next day.

Selecting the clusters is the first stage of selection, and selection of the first household of the 20 households in a cluster is the second stage. The first stage of selecting cluster sites has already been done. All cluster sites are near Zwedru town. Selection of houses in this second stage will be done by the accompanier pairs, and directions for this are included in this manual.

\section{In general, how do I administer the survey?}

For each household selected, interviewers will ask the head of each household or the primary caregiver questions about their current situation. Questions are about people in the household, sanitation, and access to health services and sensitive questions will also be asked. You should do your best to ask the questions exactly as they phrased on the questionnaire. No information on the name of those participating, or any other identifying information, will be collected.

The survey should be conducted in as much privacy as possible, avoiding locations where responses can be overheard by other persons, including other members of the household. This survey will take about 10-15 minutes for each person interviewed. You may need to encourage households to participate, but they are free to refuse to take part if they choose. There is no penalty on the household for not participating.

\section{How do I select the household to survey?}

We have already selected the areas where we will interview households. The next step is to determine where within these areas we will begin to the survey. This will be done by creating a map. The map should be turned into the TH office with the completed surveys for every cluster that is done.

1. First, you will need to draw a map of the shape of the village.

2. Next, divide the town into four areas or quadrants.

3. Number these areas 1-4.

4. Then write the numbers 1-4 on a slip of paper and fold them in half.

5. Select a slip of paper without looking.

This is an example for the village of Wala:
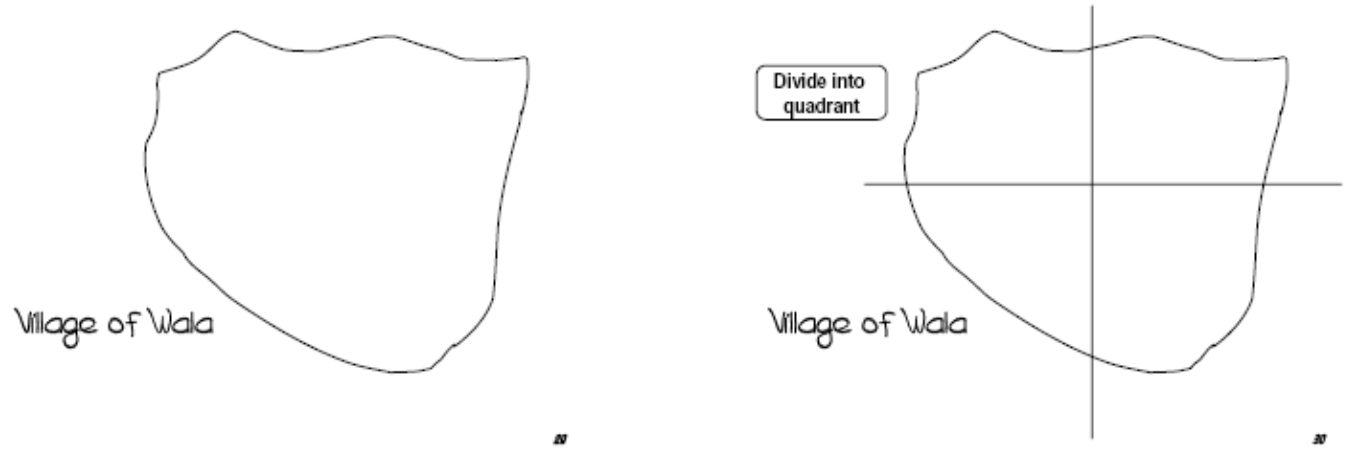


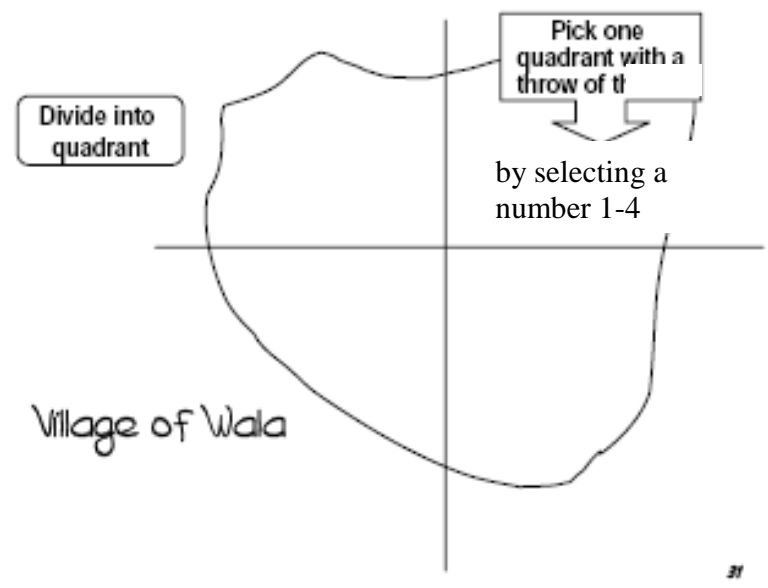

6. Next, go to the center of the village or the quadrant. This should be a location that represents the middle point of the village or the area. Please write this on the map.

7. Randomly pick a direction by spinning a pen. Indicate this direction on the map.

8. Follow the street/path and begin your survey by walking along the closest street/path near the direction chosen by the pen.

9. Interview the first house you come to on this path. Draw this household on the map.

10. When you finish interviewing a household, and are ready to leave, go to the nearest household for the next interview. This household does not have to be in the road or path; it should be the house that has the nearest front door. If nobody is home, or they decline to participate then visit the second nearest household. Indicate on the map each household that not home or where no adult is present.

11. Men and women accompaniers should interview people in the community of their same gender, if possible.

12. Repeat the process of interviewing the closest house nearby until you have surveyed 20 houses.
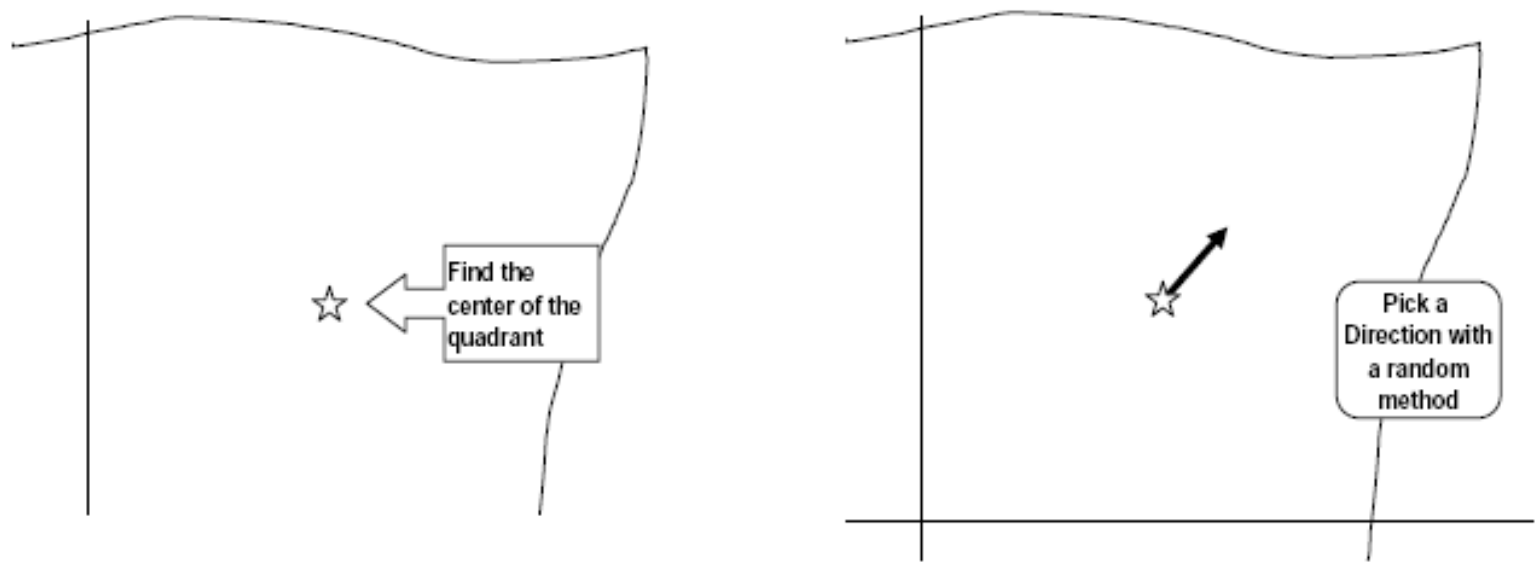


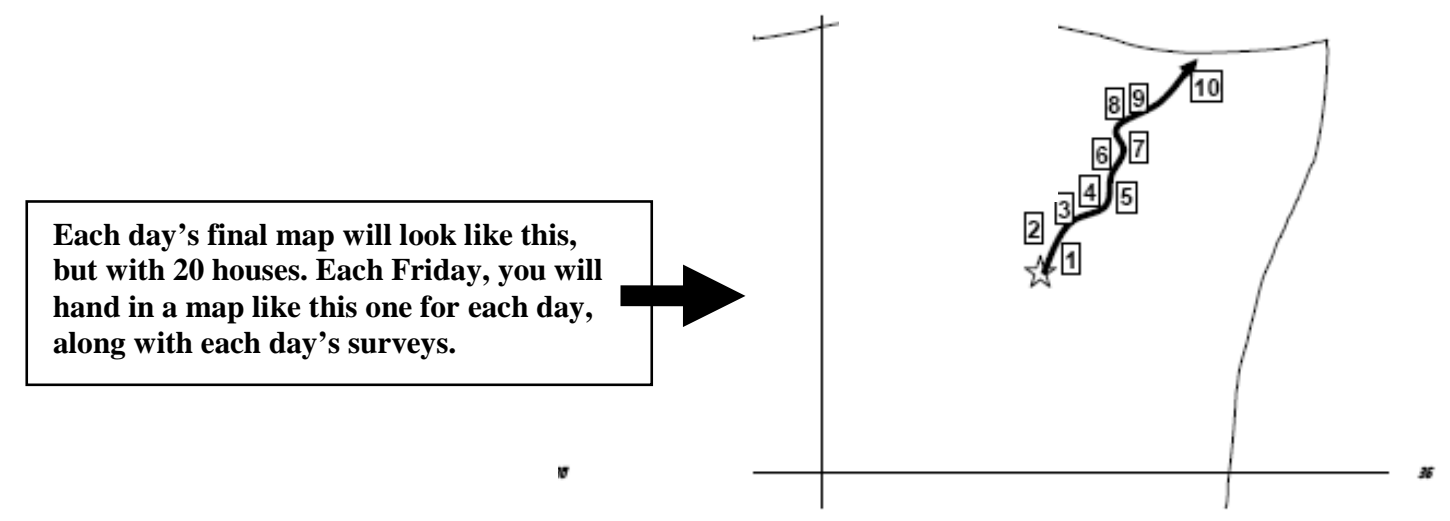

Please make sure to keep all of the maps you make with what occurred at each household that you visit. The maps should be turned in with the surveys at the end of the week.

Within a dwelling, how do I identify a household?

For a household survey it is important to define what a household is. For the purpose of this study, a household is a group of people that have slept in the same dwelling and eat out of the same pot for the last three weeks. A household does not include the occasional guest who stops in for a few days. A household may contain people are from a related family, but it also may contain other people who are not related by blood. A household does include any persons unrelated by blood, but who are sleeping and eating in the house or apartment-so a household and family are not the same. If a household consists of two or more families, (such as two brothers and their families living in the same house) and these families prepare their meals and eat together, then the oldest one should be considered the household head.

Households with Renters: in cases where the house that is selected has more than one family living there as renters of separate rooms, select only family to interview. If there are two or more families living in the same house, but one family is a renter and lives separately, these should be counted as different households. Only one family in each house should be interviewed.

\section{Once I find a household, who I interview?}

Ask to speak to the head of the household or primary caregiver. If it is a single headed household, speak with the person who is the head. If the primary caregiver or head of household is not home, you can speak with another adult in the household. If no adult is present in the household, skip this household and move to the next household. If you think that someone will be back soon in the household you skipped, you can go back to interview that person. If no one is home, or an adult will not be home soon, please continue to the next nearest household.

\section{How do I conduct the interview?}

Explain the survey to the household head primary caregiver by saying that you are conducting a survey of the health conditions around Zwedru town to find information which will improve the assistance given to their communities. Read the consent form script and secure their permission to proceed. If they do not agree, thank them and proceed to the next household. If they agree to proceed, mark "yes” on the consent form and have them initial the paper and begin the interview.

You must ensure the privacy of the people you are interviewing. Before you begin the interview, please make sure you are in a private and quiet place. If other people come around you during the interview, please ask them to leave.

In conducting the interview remember that people are concerned about revealing sensitive information, so you must put them at ease in your manner and your speaking and always show respect and treat the respondent with dignity.

\section{How do I fill out the questionnaire?}

1. Do not read the possible responses to the household head.

2. Tick the box for the response which is closest to what respondent says. Sometimes you may have to make a choice between two possible choices.

3. Sometimes a question may have to be explained at bit more to the person answering. Do as little of this explaining as possible as it may change the meaning of the question.

4. Do not lead the person to a specific response; allow them to give the answer that best suits them.

5. The respondent may not know an answer or choose not to answer a question. Make sure that they understand the question first. If they do not understand the question and do not have an answer, do not force them to answer a question they do not wish to. Questions where the answer is not known should be marked "don't know" and those where the person interviewed does not want to answer should be marked as "declined."

6. Mark the responses clearly and carefully. 
7. If for some reason the answer recorded has to be changed, draw a SINGLE line through the previous answer and enter the correct answer beside the space on in the correct box.

8. When you complete the questionnaire, continue sitting with the person being interviewed while you check through the form to be sure all the answers are completed, before you leave.

9. Please double check to make sure the Cluster and Household number are completed on both pages of the survey.

How do I finish the interview?

Once you have completed the interview and the nutrition measurement, double check the questionnaire for any missing content. If answers are missing, be sure to clarify these with the household before you go. Once you have finished checking over them form and are ready to leave the household, be sure to thank them for their time.

\section{What do I do with the forms when they are completed?}

After the forms are completed they should be returned to the TH office on Fridays. By this Friday the $15^{\text {th }}$, one accompanier pair should finish three clusters (60 households) and turn in the forms. By next Friday the $22^{\text {nd }}$, three more clusters (60 households) should be finished. All surveys and cluster maps must be turned in by Jan $22^{\text {nd }}$ Friday at the TH office.

\section{What do I turn in on Friday?}

1. the daily maps made, listing the households surveyed, households where no one was home and households that declined to participate

2. the filled out forms

Thank you!

Please call me with a missed call (or other method of calling that is no cost to the reader) at any time to answer any questions and I will call you back.

[Name of administrator]

\#\#\#\#\#

\section{Daily Checklist}

1. Travel to that day's cluster with your co-accompanier

2. Identify the first household by drawing your map, etc

3. Identify the adult to interview

4. Get consent to do the interview, and have the person initial the survey

5 . Find a private place to talk

6. Do the survey

7. Repeat the steps above for 20 households each day

Each Friday: hand in 3 maps, 60 surveys to the Tiyatien Health office in Zwedru 